\title{
Internacionalización del currículo en México desde la innovación de asignaturas en inglés
}

Internationalization of the curriculum in Mexico from the innovation of subjects in English

\section{Volumen 21, Número 2 \\ Mayo - Agosto \\ pp. 1-31}

\section{Edgar Oswaldo González Bello Isabel María García-Meza}

\section{Citar este documento según modelo APA}

González Bello, Edgar Oswaldo., y García-Meza, Isabel María. (2021). Internacionalización del currículo en México desde la innovación de asignaturas en inglés. Revista Actualidades Investigativas en Educación, 21(2), 1-31. Doi. 10.15517/aie.v21i2.46781 


\title{
Internacionalización del currículo en México desde la innovación de asignaturas en inglés \\ Internationalization of the curriculum in Mexico from the innovation of subjects in English
}

\section{Edgar Oswaldo González Bello Isabel María García-Meza²}

\begin{abstract}
Resumen: Las instituciones de educación superior evocan esfuerzos hacia acciones innovadoras que impulsen la internacionalización del currículo y promuevan el desarrollo de la competencia intercultural en sus programas educativos, a través del uso del inglés como medio de instrucción. Considerando el enfoque teórico de la innovación educativa y la perspectiva de la internacionalización, este texto tiene como objetivo analizar los factores que restringen un proceso innovador, en el cual se implementan asignaturas en la Universidad de Sonora, México desde la percepción del profesorado. Desde el enfoque cuantitativo y el método descriptivo, se realiza la aplicación de un cuestionario digital a 36 personas docentes que fueron acreditadas durante 2018-2019 para ofrecer este tipo de enseñanza. Los resultados denotan aspectos que, en su mayoría, restringen el proceso de innovación, aunque también destaca el interés y disposición de docentes hacia este proceso. Los factores que restringen comprenden la insuficiencia de la gestión institucional, al interior de instancias administrativas y de los departamentos, además de la falta de apoyo a docentes y el seguimiento a estas asignaturas, junto con la ausencia de un entendimiento compartido de los objetivos de la innovación; situación que permite suponer una comprensión programática acerca de la internacionalización. Se concluye la necesidad de promover políticas institucionales articuladas con la práctica, comprensión y adaptación a diferentes campos disciplinares, estableciendo pautas más prácticas para la implementación de este tipo de internacionalización. Además de la pertinencia de utilizar otras perspectivas teóricas en el estudio de la internacionalización del currículo.
\end{abstract}

Palabras clave: educación superior, internacionalización, innovación educativa, inglés.

\begin{abstract}
Higher education institutions evoke efforts towards innovative actions that encourage the internationalization of the curriculum and promote the development of intercultural competence with the use of English as a medium of instruction in their educational programs. Considering the theoretical approach of educational innovation and the perspective of internationalization, this text analyzes the restrictive factors that generate in an innovation process where the teaching of subjects in English is implemented for a public institution of higher education in Mexico. From the quantitative approach and the descriptive method, a digital questionnaire is applied to 36 accredited teachers during 2018-2019 to offer this type of teaching. The results indicate aspects that mostly restrict the innovation process, although the interest and willingness of teachers towards this process also stands out. The limiting factors include the insufficiency of institutional management, within administrative bodies and departments, the lack of support for teachers and follow-up to these subjects, and the absence of a shared understanding of the innovation's objectives; situation that allows to suppose a programmatic understanding about internationalization. The need to promote institutional policies articulated with practice, understanding and adaptation to the different disciplinary fields is concluded, as well as establishing more practical guidelines for the implementation of this type of internationalization. In addition to the relevance of using other theoretical perspectives for the study of the internationalization of the curriculum.
\end{abstract}

Keywords: higher education, internationalization, educational innovation, english.

\footnotetext{
1 Profesor-Investigador de tiempo completo en la Universidad de Sonora, Sonora, México. Doctor en Ciencias Sociales por la Universidad de Sonora, México. Dirección electrónica: edgar.gonzalez@unison.mx ORCID: https://orcid.org/0000-0001-6297-2516

2 Estudiante de la Maestría en Innovación Educativa y Licenciada en Enseñanza del Inglés por parte de la Universidad de Sonora, Sonora, México. Dirección electrónica: isabelgarciam.96@gmail.com ORCID: https://orcid.org/0000-0002-0535-3740
}

Artículo recibido: 30 de noviembre, 2020

Enviado a corrección: 19 de febrero, 2021

Aprobado: 22 de marzo, 2021 


\section{Introducción}

En los últimos años, el proceso de internacionalización ha llevado a que las universidades confronten diversos cambios en la búsqueda de ampliar el alcance de sus actividades y funciones académicas, más allá de sus límites nacionales (Delgado-Márquez, Hurtado-Torres y Bondar, 2011). A raíz de esto y para superar acciones delimitadas a la movilidad del alumnado y del profesorado en el extranjero, además de la firma de convenios con instituciones de otros países, se ha buscado e insistido en promover la integración del componente de internacionalización en el currículo universitario, especialmente en la enseñanza, con la integración de la dimensión intercultural (Knight, 1997).

Uno de los mayores impactos que han tenido la globalización, las exigencias de internacionalización y el aumento de la movilidad académica en las instituciones de educación superior es el uso creciente del inglés como lengua franca y lengua científica. Dado que este idioma es el medio principal de comunicación a nivel internacional (Cogo y Dewey, 2012), se ha pretendido enfocar la atención en otros aspectos, tales como su uso en ámbitos académicos y los requerimientos que van más allá del dominio lingüístico, es decir, la competencia intercultural.

A nivel mundial, el uso de EMI (English as a medium of instruction) en educación superior ha sido implementado e investigado desde finales del siglo pasado, principalmente en partes de Europa, Australia y Asia; a diferencia de México, donde esta práctica es escasamente promovida. Mientras que en lo general, el uso de EMI es abordado desde sus ventajas y aportaciones a la formación profesional, en lo particular poco se problematiza acerca de las dificultades que representa el desempeño en una segunda lengua para el personal docente y sus estudiantes (Jenkins, 2014). Si bien, se tiene conocimiento de que esta acción, como tipo de enseñanza, viene siendo implementada en algunas universidades públicas del país, es limitado lo que se ha estudiado, en especial, desde la perspectiva docente.

Con el conocimiento de que la integración de la dimensión internacional e intercultural en la enseñanza universitaria se logra mediante la oferta de programas y/o asignaturas en inglés, diversas instituciones han retomado esta propuesta como una estrategia innovadora para avanzar en la internacionalización del currículo. Si el objetivo de distintas estrategias institucionales de internacionalización lleva a la introducción de algo nuevo (dimensión internacional e intercultural), en la institución, sería conveniente centrarse también en los procesos de cambio relacionados con los objetivos y estrategias, e investigar qué factores determinan su éxito y/o fracaso (Van der Wende, 1999). 
En México, específicamente en la Universidad de Sonora, desde el año 2017 se formuló una innovación curricular para impulsar la impartición de asignaturas en inglés en sus diferentes programas educativos. Para esto, se acreditó a docentes que demostraran un dominio suficiente del idioma mediante capacitaciones institucionales, con el propósito de que se utilizara el inglés como medio de instrucción en clases presenciales. Especialmente, con el fin de alcanzar un escalón más en la internacionalización tan deseada en el prestigio de las instituciones. De acuerdo con datos proporcionados en 2019 por la Unidad de Transparencia de la institución, entre los años 2018 y 2019 se concretó la capacitación y acreditación de 42 docentes que mostraron interés para impartir asignaturas en inglés. Esta acreditación recibida por el personal académico es un criterio de permiso o impedimento para ser programado en asignaturas de este tipo.

De entre las personas que fueron reconocidas con las posibilidades técnicas y el aval de la institución para ofrecer asignaturas en inglés, destaca su adscripción disciplinar al ser docentes de Ciencias Exactas y Naturales (31\%) e Ingenierías (29\%) los que más se interesaron en participar en la innovación, especialmente quienes imparten cursos de programas como Licenciatura en Física e Ingeniería Industrial. Fue menor la atracción para el profesorado de Ciencias Sociales (7\%) y de Económicas-Administrativas (2\%), lo cual tuvo como resultado que estas áreas de estudios tuvieran menos disponibilidad de recurso humano para este tipo de docencia.

En el sentido de intensificar el resultado de esta innovación, la misma institución estableció como una de sus metas un número ascendente de asignaturas en inglés a impartirse año con año y con la expectativa de alcanzar 120 cursos para 2021; no obstante, entre 2018 y 2019 se ha mantenido la oferta de cinco asignaturas impartidas en inglés en promedio por semestre. Si se considera que la institución ofrece 49 programas de licenciatura y 47 a nivel posgrado, compuestos por una cantidad mucho mayor de cursos, con un personal académico de tiempo completo de 1029 sujetos, es poco alentador que en cuatro años se logró una acreditación limitada de profesoras y profesores. En el año 2019 destaca que el 81\% de este grupo académico acreditado no impartió ningún curso en inglés, a pesar de contar con la acreditación para hacerlo. Esto permite suponer que los propósitos definidos difícilmente podrán alcanzarse, al menos, en el tiempo establecido.

Así, este panorama se vuelve más complejo y exigente para la Universidad de Sonora, ya que, al valorar la relevancia del dominio de este idioma, la institución aprobó en 2019 una normativa denominada Lineamientos para el Componente Curricular, donde se estableció, de 
manera obligatoria, la inclusión de un 15\% de asignaturas en dos idiomas (español e inglés) para todos los programas de estudios de nivel licenciatura.

Estas acciones denotan que la impartición de asignaturas en inglés en la universidad representa una innovación curricular, puesto que se busca transformar los procesos de enseñanza-aprendizaje y, por ende, se busca alterar la práctica docente (Casanova, 2015). Entre los componentes de esta innovación (De la Torre, 1997) destacan, primeramente, su nivel de cambio, el cual implica una alteración de comportamiento a nivel colectivo, puesto que se ha buscado una transformación de la enseñanza en el profesorado con los cursos de capacitación/habilitación.

En ese sentido y por los elementos que son objeto de cambio, como el contenido, metodología, materiales y actividades, se trata de llevar a cabo una innovación curricular estratégica con la implementación de asignaturas en inglés. Dichos objetos apuntan a una reestructuración como modo de cambio, puesto que son los mismos programas de la universidad que sufren la alteración al idioma inglés. Además, la enseñanza con el idioma inglés representa una meso-innovación, es decir, conlleva transformaciones a nivel institucional, en el aula y, se espera que atañe a todos los departamentos y disciplinas. Dichas transformaciones buscan promover la internacionalización que son impulsadas por instancias administrativas de la universidad.

Si bien, parte del personal docente ha mostrado su interés en participar, motivo por el cual destaca haber logrado su acreditación, algo sucede entre el transcurso de esta habilitación y la implementación de la innovación en el aula. Resulta indispensable mediar entre los niveles meso (órganos rectores escolares) y micro (aula) porque, al coincidir con Díaz-Barriga (2010), se puede suponer que el profesorado queda atrapado en este proceso de apropiación de la innovación, posiblemente determinado por el surgimiento de problemas que se manifiestan entre las políticas que se buscan implementar en una institución y su práctica real en el aula.

Además, se pone en discusión cómo la implementación de esta innovación implica cambios en la práctica, pero que, además, estos varían significativamente según los campos disciplinares, en los cuales se aprecia una disparidad en el personal académico que ha sido acreditado, lo cual también se refleja en el reducido número de profesoras y profesores que imparten asignaturas en inglés. De la misma manera, se cuestionan los diferentes significados y valores que otorgan los grupos de académicos desde sus diferentes disciplinas, así como su reacción frente a este particular proceso de internacionalizar el currículo mediante la 
innovación de la enseñanza con el inglés como medio de instrucción. En esto, destacan las múltiples complejidades del idioma, relacionadas con su utilidad para fines académicos, así como el desconocimiento de que tanto el alumnado como el profesorado posiblemente tengan carencias de rasgos de una competencia intercultural.

Esto sustenta la pertinencia de indagar este proceso particular de innovación que, en su fase de implementación, demanda considerar aquellos factores que restringen y representan un obstáculo para la innovación, los cuales van desde la propia naturaleza del proceso y motivos personales, hasta restricciones de tipo organizativas y contextuales (Rivas, 2000). Una innovación, como lo plantea Escudero (2014), debe basarse en los sentidos, significados y experiencias que tiene el personal académico sobre lo que implica este proceso de innovación de asignaturas en inglés. Aunque existen múltiples esfuerzos por parte de las universidades para implantar programas impartidos en otro idioma, con la visión de generar la internacionalización que se espera en la educación superior, los cambios que implica una innovación de este tipo son, por demás, complejos, máxime por los efectos que tiene en cada docente y la variación de percepciones en el transcurso de su implementación (Bedenlier y Zawacki-Richter, 2015).

Así, el proceso de internacionalización del currículo en la Universidad de Sonora (México), desde la innovación de asignaturas en inglés, permite asumir ciertas dificultades que pueden ser reflejadas en asuntos que restringen este proceso. De entrada, aquellos aspectos que refieren al apoyo y la atención de necesidades del profesorado, además de los requerimientos para la gestión institucional que demandan estas asignaturas.

Si las universidades buscan implantar acciones de internacionalización que incidan en el currículo y logren el desarrollo de la competencia internacional e intercultural en el alumnado, convendría generar políticas y programas que contemplen los objetivos de cada una de las disciplinas. También, efectuar acciones de seguimiento con el personal académico y considerar las implicaciones que se derivan de estas intenciones. Debido a la ausencia de metas explícitamente formuladas y a la falta de comprensión de la internacionalización, desde una perspectiva pedagógica, en torno a las experiencias sobre el aprendizaje y sus condiciones en los diferentes contextos que guarda cada disciplina (Wihlborg, 2009), se sustenta la pertinencia de analizar los procesos innovadores que guarden relación con dichos aspectos.

Conocer las diversas formas en que se entiende y expresa la internacionalización en todas las disciplinas e instituciones contribuye a informar la planificación estratégica de todo 
un campus, especialmente en lo que se refiere a la internacionalización del currículo (Agnew, 2013). Así, explorar la percepción que se tiene de una innovación, la cual busca impulsar la internacionalización, permitirá comprender la diversidad de sus impactos en la identidad, orientación y experiencia en una variedad de contextos particulares dentro de la institución (Turner y Robson, 2007). Esto puede revelar el grado en que se percibe a la internacionalización como un proceso más manejable. Un análisis de este tipo podría ayudar y orientar a las personas encargadas de la formulación de políticas y a administradores de la institución a encontrar y desarrollar soluciones a los desafíos encontrados, y considerar los posibles factores que podrían facilitar la implementación de la innovación (Cai, 2017).

Este texto tiene como objetivo principal analizar los factores (organizativos, contextuales y personales) que restringen el proceso de innovación en el cual se implementan asignaturas en inglés para una institución pública de educación superior en México, desde la percepción de las personas docentes. Para esto, se toma como base el enfoque teórico de la innovación educativa y los márgenes de la perspectiva de internacionalización del currículo con el uso de inglés como medio de instrucción.

\section{Referente teórico}

El tema de la internacionalización ha sido investigado desde distintas perspectivas y la literatura que aborda este proceso es abundante. La internacionalización es un componente innegable en las instituciones de educación superior, al grado de intensificarse cada vez más los esfuerzos para implantar este proceso en las universidades (Delgado-Márquez et al., 2011). Esto ha sido argumentado por investigaciones que abordan la internacionalización en educación superior (Knight, 1994; Knight y de Wit, 1995; De Wit, 2002; Bedenlier y ZawackiRichter, 2015; Khan, Omrane y Bank, 2016) y destacan que, a grandes rasgos, este proceso consiste en integrar las dimensiones internacional e intercultural en la enseñanza.

Como lo plantea Friesen (2012), el proceso de internacionalización en este nivel educativo puede ser entendido desde dos puntos de vista diferentes: por un lado, desde una comprensión programática como las expuestas en documentos institucionales, con las formas en que las universidades buscan desarrollar una mayor presencia en el mercado internacional. Por otro lado, desde una perspectiva más individual, la internacionalización tiene el fin de alcanzar procesos educativos que promueven la conciencia, comprensión e inclusión de diferentes culturas. Una de las estrategias que pretende tener impacto en dichos procesos es la internacionalización en casa, la cual busca generar cambios en el currículo, los programas, 
las asignaturas, en los procesos de enseñanza-aprendizaje y en las actividades extracurriculares (Knight, 2008).

Por esta misma razón, Beelen (2011) puntualiza que, entre las políticas y actividades que buscan impulsar la internacionalización en casa en el contexto de América Latina y el Caribe, destacan el deseo de fortalecer el contenido internacional/intercultural del currículo y la enseñanza de lenguas extranjeras como parte del plan de estudios. No obstante, de manera contradictoria, reporta que estas actividades son las que menor atención, apoyo y recursos reciben, a pesar de las políticas que buscan favorecer la internacionalización y la enseñanza de idiomas en los últimos años (Didou, 2017).

Resalta que las políticas que buscan integrar la internacionalización, como lo muestra Didou (2006), han sido enfocadas principalmente a programas de movilidad internacional del alumnado y del profesorado. Sin embargo, estos esfuerzos también deben ser planteados con el objetivo de mejorar la calidad de la educación y la innovación del currículo, con el fin de complementar los contenidos de los programas académicos, sus asignaturas y los métodos de enseñanza (González, 2016).

De esta forma, la estrategia de internacionalizar el currículo es uno de los aspectos que constituyen la internacionalización en casa, tal como lo plantea Knight (2008). Este concepto es definido por Nilsson (2003) como el "brindar conocimientos y habilidades internacionales e interculturales, con el objetivo de preparar a las y los estudiantes para actuar (de manera profesional, social y emocional) en un contexto internacional y multicultural" (p. 31). En general, integrar la perspectiva de internacionalización en un currículo refiere a la capacidad de incorporarla a nivel de políticas académicas, políticas curriculares, de diseño de programas y a nivel aula (Van der Wende, 1996). Entre sus metas principales está lograr el mejoramiento de idiomas extranjeros, la comprensión integral de características de diferentes países y culturas para el desarrollo de la competencia intercultural.

En este sentido, la dimensión intercultural se advierte como un punto clave para la internacionalización en educación superior. La integración de esta a los planes y programas de estudios es el resultado de la internacionalización de mercados y la globalización cultural con necesidades de intercambios con personas de distintos países (Paricio, 2014). Dichas necesidades hacen que el aprendizaje de un idioma sea una de las principales formas de generar competencia intercultural, lo cual va más allá de la concepción instrumental del aprendizaje de este. Se fundamenta que un idioma no puede ser concebido como parte de la cultura, sino que, a través de este, se expresan las prácticas y creencias de una cultura. Por 
ende, el aprendizaje de un idioma trasciende el marco lingüístico e implica una formación más integral, donde también se desarrollan otras competencias.

Si bien, una de las formas que han adoptado las universidades para brindar un currículo internacionalizado es con la oferta de programas y asignaturas impartidas en una lengua extranjera; Leask (2001) describe que esta es solo una de las características que posee un currículo internacionalizado dentro de una gama de posibilidades que es más amplia. Sin embargo, los programas ofertados en lengua extranjera deben también abordar problemas de comunicación, para así ofrecer al alumnado un desarrollo en competencias (internacional e intercultural). La implementación de este tipo de programas representa, o debería representar, una acción planificada que implica producir un cambio en las instituciones educativas, tanto en su cultura, estructura, políticas y metodologías, como en sus productos y procesos organizativos. Motivo por el cual puede ser considerado como un proceso de innovación educativa (De la Torre, 1997; Tejada, 1998; De la Herrán y Paredes, 2012).

En el contexto de la internacionalización, Van der Wende (1999) distingue diversos tipos de innovaciones, mismos que analiza y caracteriza según los cambios que intentan generar. En el caso de la Universidad de Sonora (México), se observan esfuerzos por realizar cambios holísticos dentro de sus organizaciones. Es decir, la adopción de una innovación a nivel institucional con un propósito compartido y cambios ambiciosos en funciones, tales como la investigación, docencia y servicios universitarios. Las innovaciones que impliquen cambios a tal magnitud, son las más difíciles de adoptar y las que más obstáculos atañe para una adopción exitosa.

Como lo presenta Leask (2001), al incursionar en la internacionalización del currículo a través de programas/asignaturas en inglés es indispensable evitar la concepción errónea de considerar al currículo como internacional por el hecho de ofrecerse en este idioma. Más allá de esto, es manejar de manera explícita cómo los programas/asignaturas incluyen en sus objetivos, contenidos y evaluación el desarrollo de comunicación y habilidades interculturales. Esto, analizado desde la perspectiva de la innovación educativa, se puede considerar como un proceso de innovación curricular (Casanova, 2015), puesto que esta forma de internacionalización busca introducir otras formas de actuación docente, métodos y estrategias de enseñanza. De modo que, para la enseñanza en inglés es necesario que la comunidad docente lleve a cabo cambios en su práctica, su programación, recursos, modelos de actividades, todo cuanto llega al estudiante en su proceso de aprendizaje, en función del idioma y la inclusión del componente intercultural en una asignatura. 
Diferentes investigaciones (Coleman, 2006; Smit y Dafouz, 2012; Marsh, PavónVázquez y Frigols-Martín, 2013; Wächter y Mainworm, 2014; Bradford, 2012) han plasmado cómo el idioma inglés se ha adoptado como medio de instrucción, en diversas universidades, al considerarlo como facilitador de intercambio académico global que promueve el desarrollo del conocimiento científico y el avance profesional. En partes de Asia, Australia y América Latina, Nikula (2016) presenta cómo se comienza a adoptar este tipo de enseñanza bajo la etiqueta de CLIL (Content and Language Integrated Learning), el cual se asemeja al enfoque de inmersión utilizado en América del Norte, donde parte de un plan de estudios se enseña a través de una segunda lengua extranjera. El enfoque CLIL, que integra el aprendizaje de contenido e idiomas, no solo se centra en el dominio instrumental del idioma, sino también en términos de comunicación y cognición, promoviendo así conocimiento y competencia intercultural (Chostelidou y Griva, 2014).

Derivado de las intenciones anteriores, algunas investigaciones (Wächter y Mainworm, 2014; Bradford, 2012) evidencian que, entre los obstáculos más comunes al implementar programas en inglés, destaca el nivel bajo de dominio del idioma por parte del profesorado y del alumnado, también limitadas habilidades lingüísticas, lo cual genera la resistencia a enseñar y aprender en otras lenguas. Se suma una formación docente que resulta inadecuada o insuficiente, problemas a nivel organizativo y administrativos dentro de las universidades, la falta de interés por parte de las y los estudiantes hacia dichos programas, entre otros. Además, los intereses de las instituciones y las exigencias de las diferentes áreas disciplinares también determinan y dificultan la implementación de estas iniciativas de internacionalización, incluso se consideran como incompatibles con ciertas disciplinas.

En este mismo sentido, Cai (2017) expone que se generan diferentes puntos de vista sobre el proceso de innovación y considera más susceptible la internacionalización con ciertas disciplinas, a diferencia de otras y dentro de estas, pueden existir diferentes perspectivas sobre el proceso de internacionalizar el currículo (Bell, 2004). Este proceso, como lo presenta Clifford (2009), genera debate académico y respuestas variadas dentro de las disciplinas, debido a que sus diferencias también se encuentran en la enseñanza y la investigación, además de la relación entre profesorado y alumnado. Se argumenta sobre la pertinencia de explorar las diferentes respuestas disciplinarias al proceso de internacionalización del currículo a través de la categorización de Becher (1989), para tratar de identificar y comprender, a su vez, las diferentes resistencias y formas de avanzar en este proceso a través de la variedad de disciplinas. Por esto, conviene que se establezcan pautas más prácticas para la 
implementación de procesos de internacionalización en el currículo, donde se atiendan las preocupaciones, dificultades e incertidumbres del personal académico involucrado (Osakwe, 2017).

En esa lógica, Casanova (2015) resalta que los procesos de cambio son progresivos y se producen mediante la implementación de innovaciones, planeadas y estructuradas en fases, tales como el reconocimiento de la necesidad de cambio, formulación de soluciones, implementación de planes e institucionalización, es decir, la adopción de la innovación. La primera etapa de reconocimiento, como lo expone Van der Wende (1999), se logra desde la importancia que las instituciones otorgan a la internacionalización, a pesar de que con frecuencia los compromisos y actividades emprendidas no sean del todo claras. A pesar de esto, la formulación de innovaciones, como la enseñanza en inglés, comienzan a implementarse. Además, impera la creencia de que la fase de implementación es la conclusión de un proceso de innovación, cuando la institucionalización es la fase más crítica debido a que la internacionalizacion ha sido mayormente una respuesta reactiva, en lugar de proactiva, por parte de las instituciones de educación superior. Esta situación indica la necesidad de una mayor comprensión sobre cómo y por qué fallan las innovaciones que buscan impulsar la internacionalizacion en las universidades.

Las dificultades de la implementación y la calidad de estos programas/asignaturas en inglés, persisten a medida que Bradford (2012) enlista retos que surgen en el proceso, como los relacionados con el idioma, la cultura y la estructura de los programas, así como limitación en la competencia lingüística del profesorado y del alumnado. Entre los retos culturales, la falta de conocimiento intercultural del profesorado es una limitante y se advierte que los desafíos lingüísticos y culturales aumentan su carga de trabajo. Además, la falta de apoyo a docentes por parte de instancias administrativas y la escasa efectividad de la gestión institucional con respecto a estas iniciativas, representan más desafíos por superar. Para una mejor implementación de la enseñanza con el inglés como medio de instrucción, es pertinente la atención de tres desafíos: lingüísticos, culturales y organizacionales.

También, a nivel de diseño de políticas institucionales para internacionalizar un currículo, diversos estudios (Green y Whitsed, 2013; McKinnon, Hammond y Foster, 2017, Arango y Acuña, 2018) establecen que la dificultad comienza desde la confusión que existe alrededor del concepto. Con frecuencia es malinterpretado o implementado como una serie de acciones aisladas, sin establecer estrategias ni procesos evaluativos para determinar el nivel de avance del proceso. De manera que, una revisión constante de la innovación es crucial para una 
implementación y adopción eficaz, donde se atiendan las preocupaciones, dificultades e incertidumbres de las y los docentes.

Otros trabajos en América Latina (McDougald, 2015; Corrales, Paba y Santiago, 2016) muestran que es limitada la implementación del enfoque EMI/CLIL en educación superior. Desde una perspectiva académica, se manifiesta el beneficio de este enfoque para el desarrollo de habilidades del lenguaje y del contenido en el alumnado. Sin embargo, aún se requieren objetivos claros y trabajo estratégico en la implementación. En México, Núñez Asomoza (2015) reporta el potencial de la implementación de programas CLIL y, a la vez, destaca áreas de oportunidad en el personal académico y el diseño de materiales, también en los asuntos administrativos y la gestión institucional donde se advierte la necesidad de un cambio.

Asimismo, el problema para muchas innovaciones reside en que siguen una lógica unidireccional, dictada por las instituciones, y que, en esta ocasión, se refleja por la necesidad de incorporar el componente de internacionalización en el currículo. Mientras tanto, poco se reflexiona sobre las dificultades que tienen las personas encargadas de llevar la innovación curricular (enseñanza en inglés) a la práctica (Díaz-Barriga, 2010).

A partir de los aportes de Rivas (2000), se encuentra que poco se analiza el tipo y el contenido de la innovación, de modo que se sigue un procedimiento analítico para identificar los atributos que caracterizan al proceso de innovación con las asignaturas en inglés, desde la percepción docente. Los atributos son condicionantes para la incorporación de la innovación a los procesos educativos y predictores de su curso. Todo proceso innovador tiene una serie de atributos (Van der Wende, 1999; Rivas, 2000) que determinan, en gran medida, su institucionalización:

- Claridad: facilidad con que sus objetivos, estructura y características son comprendidos por los innovadores involucrados.

- Complejidad: grado en que la innovación se percibe como operativamente fácil o difícil en cuanto a capacidades requeridas para llevarla a cabo, así como intensidad y extensión de las alteraciones que la innovación implique.

- Compatibilidad: grado en que las normas, valores y objetivos de la innovación son congruentes con las organizaciones de la institución, al existir diferencias en las prácticas internacionales entre las disciplinas.

- Rentabilidad: eficacia de una innovación para satisfacer las necesidades existentes. Varía dentro de las diferentes organizaciones al ser una medida de satisfacción, lo cual 
lo hace un factor crítico para la institucionalización; resulta vital establecer vínculos de comunicación interna para conocer las necesidades de los innovadores. Aunque los programas de internacionalización distan de adaptarse a las necesidades individuales (y disciplinares), los servicios de las universidades sí pueden hacerlo.

- Beneficio potencial: el indicador más directo de la calidad o eficacia de la innovación en sí; tiene varias manifestaciones, como solución a un problema, satisfacción de una necesidad sentida por un grupo, entre otras.

La indagación en el proceso de innovación de asignaturas en inglés, a partir de los factores (organizativos, contextuales y personales) que restringen su implementación eficaz como dimensión de estudio, permite identificar sus atributos para comprender el rumbo que sigue la innovación y advertir sobre sus posibles rutas de mejora.

\section{Metodología}

\subsection{Enfoque}

Se realizó un análisis cuantitativo no experimental, de tipo descriptivo y transversal, mediante una encuesta aplicada al profesorado con características determinadas. Esto con el fin de describir la realidad y analizar relaciones, mediante la recolección de datos en un solo momento (Rodríguez y Valldeorriola, 2009). La investigación fue descriptiva, se indagó y midió la percepción docente en torno al proceso de innovación enfocado en aspectos que lo restringen. Tal como señala McMillan y Schumacher (2005), este tipo de investigación resulta apropiada al proporcionar datos valiosos a través de la descripción de actitudes, comportamientos y otras características del grupo de sujetos a estudiar.

\subsection{Unidades de análisis}

Desde la perspectiva teórica de innovación educativa y el uso del inglés en la enseñanza para la internacionalización del currículo, se indagaron distintas variables como unidades de análisis. Entre estas destacan: los motivos de participación en el proceso de innovación, la formación de las y los docentes para la enseñanza en inglés, además de los factores que impulsan y restringen este proceso de innovación. Además, otros aspectos como el perfil docente compuesto por diferentes variables independientes que permitieron efectuar otros tipos de análisis. Se realizó una categorización con la variable disciplina, a partir de las tipologías de Becher (1989): ciencias duras y ciencias blandas. Además, otra categorización 
con la frecuencia en que el personal académico ha participado en la innovación: los que han impartido una o más asignaturas en inglés y quienes aún no han impartido, a pesar de contar con la acreditación para hacerlo. Para fines de este texto, se retoman, principalmente, los factores que restringen el proceso de innovación analizados en función de otras variables.

El análisis del objeto de estudio fue en el contexto de la Universidad de Sonora, institución pública de educación superior en México, en el periodo de septiembre y octubre de 2020. Como sujetos, se consideró al total del profesorado que ha sido acreditado formalmente para impartir asignaturas en inglés. Esta población está representada por un total de 42 docentes, 27 hombres y 15 mujeres, adscritos en alguna de las seis divisiones que administrativamente conforman la institución y representan las áreas académicas. Finalmente, el proceso de levantamiento de datos obtuvo las respuestas de 36 docentes ( $85 \%$ del total) con representatividad de todas las divisiones administrativas, constituyéndose como un acercamiento inicial que permitió destacar los principales factores que restringen la puesta en práctica de una enseñanza que recurre al uso del idioma inglés, comprendida como una innovación curricular.

\subsection{Técnicas de recolección}

Para la recolección de datos, se optó por la técnica de encuesta mediante la aplicación de un cuestionario al personal académico, ya que permite recuperar aspectos de la población estudiada con el fin de producir datos cuantitativos para su análisis y tratamiento estadístico (Fàbregues et al., 2016). En función de esto, se elaboró un cuestionario digital (http://mie.uson.mx/internacionalizacion/) titulado Iniciativa innovadora de asignaturas impartidas en idioma inglés para la internacionalización del currículo, el cual fue enviado mediante correo electrónico y se informó sobre la participación voluntaria. Además, se indicó que la información proporcionada sería anónima, confidencial, reportada de manera agrupada y utilizada con fines académicos, a manera de consentimiento informado. Esto tuvo como propósito recuperar las percepciones del profesorado en torno al proceso de innovación (ver Anexo 1) a partir de diferentes unidades de análisis.

Los reactivos utilizados en el instrumento se categorizaron en diferentes tópicos y fueron de tipo formato cerrado para obtención de información demográfica y datos fácilmente clasificables (McMillan y Schumacher, 2005). Se optó por este tipo de reactivo ya que permite valoraciones exactas de percepciones y opiniones. Con excepción de una sección donde se solicita información sobre el perfil del docente, los reactivos restantes contienen tópicos que 
se responden mediante opciones en una escala Likert de cinco niveles de respuesta para medir el grado de acuerdo de los participantes. La recolección de datos se hizo mediante un envío masivo del cuestionario digital por correo electrónico.

\subsection{Procesamiento de análisis}

Se creó una base de datos con las respuestas de los cuestionarios; para el análisis de datos, se utilizó el programa SPSS 24 (Statistical Package for the Social Sciences). Como parte del procesamiento, ciertas variables que sobrepasaban los tres niveles de respuesta se agruparon en rangos con el fin de generar una mejor distribución más representativa y visual de los datos, lo cual permite hacer otras comparaciones. Asimismo, se realizó la categorización de respuestas obtenidas en reactivos de tipo abierto, donde se agruparon con fines de análisis.

\section{Resultados}

Para responder al objetivo general, los resultados referentes a factores que restringen la implementación de una innovación (asignaturas en inglés) son expuestos en primera instancia por tipo de disciplina. Inicialmente, se indagaron aspectos que restringen/obstaculizan la implementación eficiente de una innovación que refiere a la enseñanza con el uso del inglés, específicamente en aspectos de clase. En consecuencia, los primeros hallazgos denotan que existen suficientes recursos en el idioma, lo cual se aleja de ser una limitante para llevar la innovación a la práctica; el 90\% de docentes pertenecientes a disciplinas de ciencias duras señalan su acuerdo, mientras que en ciencias blandas el porcentaje es un poco menor (71\%).

Por otra parte, y en contraste, expresan mayor acuerdo con percibir una insuficiencia del idioma (ver Figura 1) para este tipo de asignaturas por parte del alumnado adscrito a disciplinas de ciencias blandas (57\%). 
Figura 1

Porcentaje de acuerdo del profesorado sobre la insuficiencia del nivel de idioma del estudiante por tipos de disciplinas, Universidad de Sonora- México, 2020

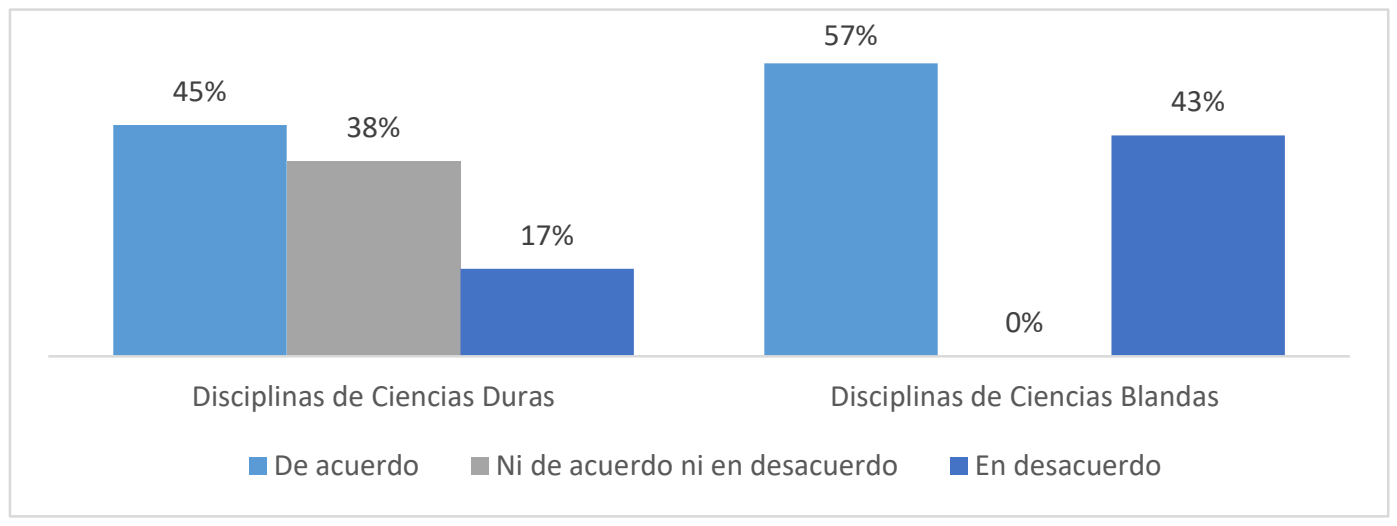

Fuente: Elaboración propia.

Con referencia a las implicaciones personales que restringen la innovación (ver Figura 2), se ubicaron diferencias importantes, principalmente con docentes que a pesar de tener la acreditación no han impartido asignaturas en inglés. En otros aspectos, como la necesidad de apoyo y retroalimentación en la práctica, o de mejorar las habilidades de adaptación de contenidos, resalta que el grado de acuerdo con dichas afirmaciones disminuye conforme la participación del profesorado impartiendo asignaturas en inglés aumenta. Esto advierte que, en primera instancia, quienes requieren más apoyo y esfuerzos institucionales para promover este tipo de innovación, son quienes están acreditados y que aún no han impartido clases en esta modalidad. Sin embargo, también destaca que un porcentaje considerable de docentes que han impartido una o más veces en inglés, expresa la necesidad de retroalimentación de su práctica y mejora de sus habilidades de adaptación. 
Figura 2

Porcentaje de acuerdo del profesorado sobre la falta de apoyo e insuficiencia de habilidades de adaptación por asignaturas impartidas, Universidad de Sonora- México, 2020

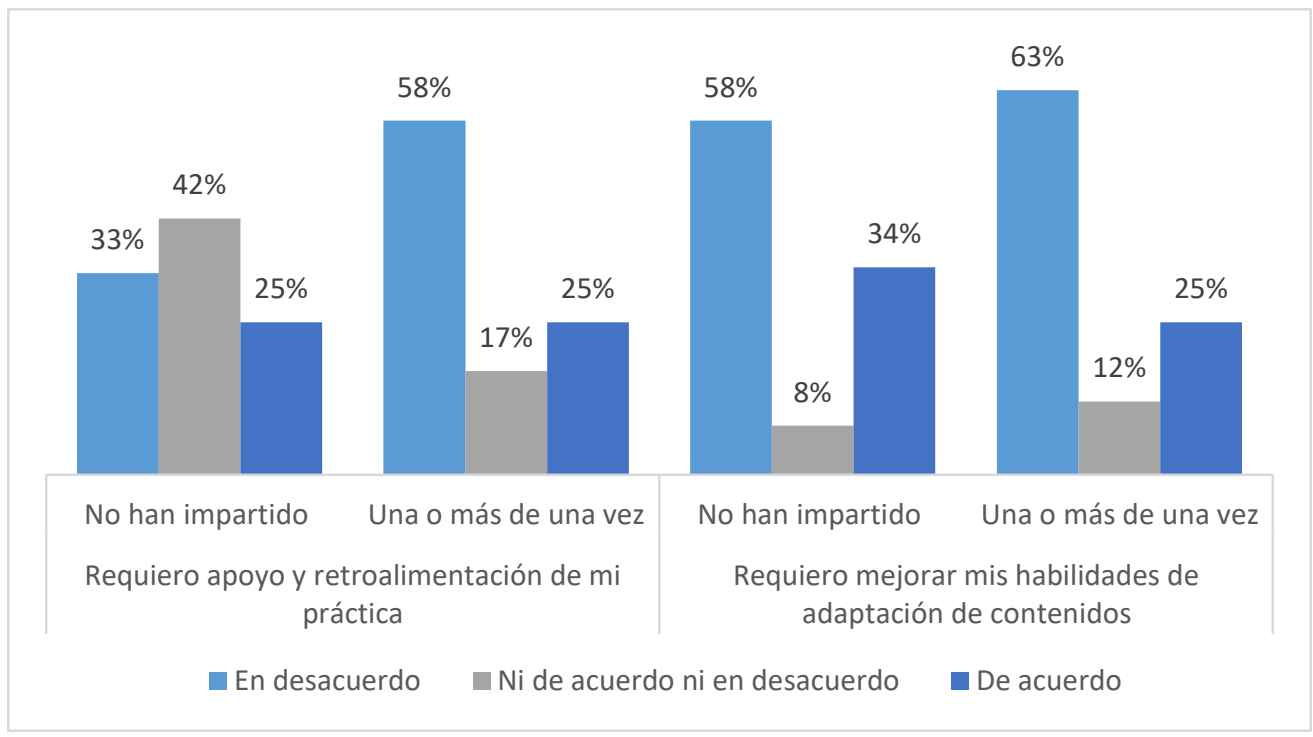

Fuente: Elaboración propia.

En cuanto a aspectos de nivel aula, son menores los elementos desfavorecedores que se advierten y, por lo tanto, se descarta su consideración como obstáculos del proceso de innovación, lo cual permite suponer que en el contexto aula el profesorado asimila los retos que enfrentan. A pesar de esto, valdría la pena profundizar en el apoyo a docentes de áreas disciplinares blandas, especialmente en la adaptación de materiales/recursos según las exigencias de las disciplinas y el idioma. Además, aunque no es excesivamente alto el grado de acuerdo con que el nivel de idioma de estudiantes para este tipo de asignaturas es insuficiente, es provechoso tomarlo como un área de oportunidad, especialmente en disciplinas de ciencias blandas, al asumir que, como señalan Corrales et al. (2016), el nivel de idioma es clave al emplearse EMI en universidades, por lo cual se requiere dar seguimiento a las habilidades lingüísticas de estudiantes.

En otro de los aspectos que refieren a la necesidad de aprender a trabajar la competencia intercultural en clase (ver Figura 3), se encuentra que, si bien la mitad de ambas poblaciones se encuentra en desacuerdo, también se visualiza que una parte importante del profesorado manifiesta la necesidad de aprender a trabajar esta competencia. Además, poco más de la mitad del personal académico acreditado que no ha impartido asignaturas en inglés, expresa estar de acuerdo con que estas asignaturas incrementan su carga de trabajo, lo cual podría relacionarse con evitar su participación. 
Figura 3

Porcentaje de acuerdo del profesorado sobre el aumento en la carga laboral y el trabajo de la competencia intercultural por asignaturas impartidas, Universidad de Sonora- México, 2020

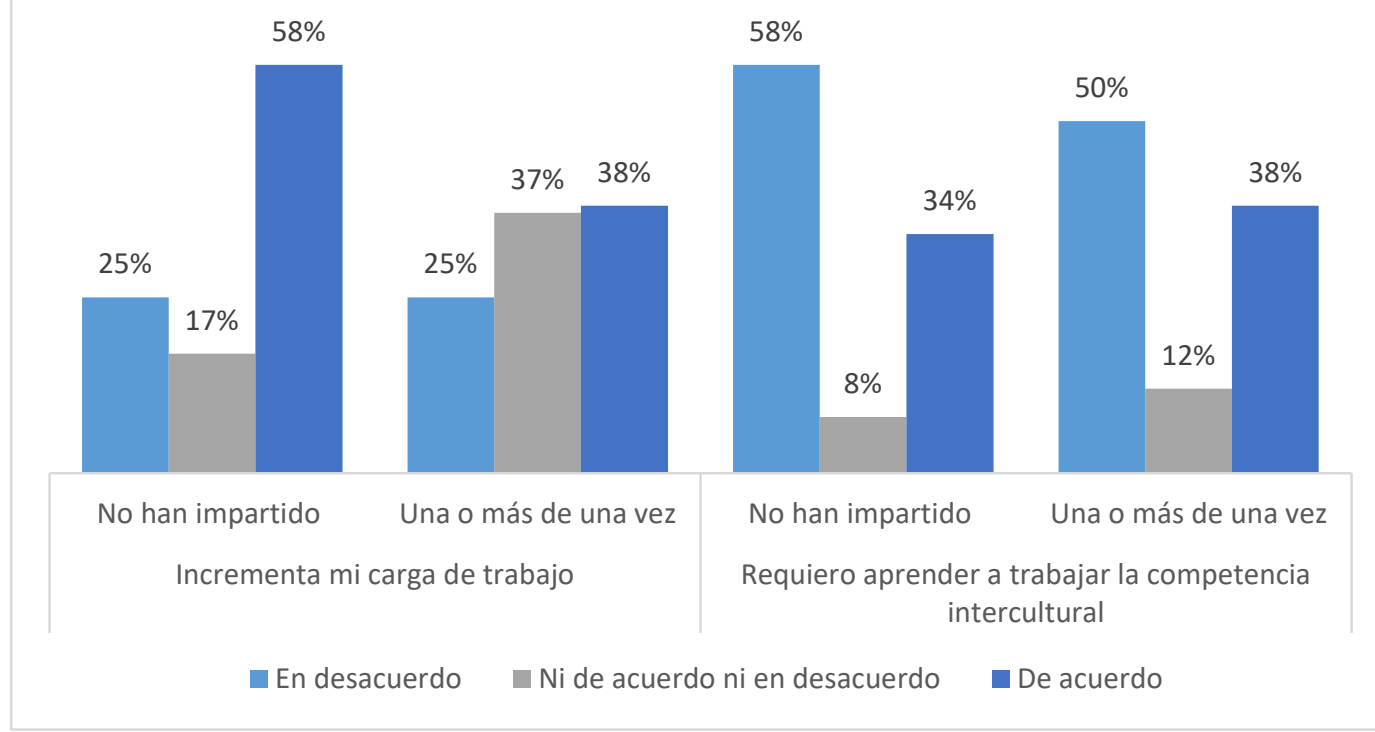

Fuente: Elaboración propia.

Aunque no se encontró un alto grado de acuerdo con que los aspectos de tipo personal sean un obstáculo en la implementación de asignaturas en inglés, se distingue que una porción importante del profesorado (tanto quienes han impartido y quienes no) reconoce la necesidad de aprender a trabajar la competencia intercultural en sus clases. Así, esta distinción entre docentes que ya han impartido asignaturas en inglés y quienes no lo hacen, permitió reconocer que esta última categorización de profesoras y profesores es quien más necesitan apoyo referente a cómo trabajar la competencia intercultural, al igual que mejorar sus habilidades en la adaptación de contenidos considerando el idioma; también porque reconocen que es mayor la carga de trabajo al involucrarse con este tipo de asignaturas.

A su vez, la participación limitada del profesorado acreditado y más de quienes aún no imparten una asignatura en inglés, puede comprenderse desde lo que expone Dunne (2011) y McKinnon et al. (2017), quienes sustentan que es común la falta de confianza del personal académico sobre su propia habilidad, resaltando la falta de respaldo y a veces de la preparación insuficiente para participar en este tipo de internacionalización curricular que alude a actividades en el aula.

Esta situación no implica que no requiera apoyo el profesorado que ha impartido más de una asignatura en esta modalidad, puesto que muchas veces se carece del apoyo institucional para crear currículos internacionalizados y se ven en la necesidad de improvisar 
(Arango y Acuña, 2018). En consecuencia, es probable que la implementación de este tipo de asignaturas se aleje de resultados favorecedores, más si se olvida la necesidad de articular la innovación con una práctica más eficaz y una comprensión compartida de sus principios en las diferentes disciplinas (Coyle, 2010).

Al analizar las implicaciones negativas de tipo institucional según diferencias disciplinares (ver Figura 4), resalta que ambos tipos de ciencias -duras y blandas- no están de acuerdo en que las asignaturas en inglés no tengan congruencia con sus objetivos disciplinares. Esto evidencia la relevancia que otorga el profesorado a este tipo de asignaturas. Por otro lado, se encontraron diferencias porcentuales entre las disciplinas en cuanto a la ausencia de orientaciones prácticas en las capacitaciones que ofrece la institución, siendo mayor el grado de desacuerdo en docentes de ciencias blandas; mientras que, en ciencias duras la percepción es más variada.

Figura 4

Porcentaje de acuerdo del profesorado sobre la ausencia de orientaciones prácticas en capacitaciones e incongruencia de materias con objetivos disciplinares por tipos de disciplinas, Universidad de Sonora- México, 2020

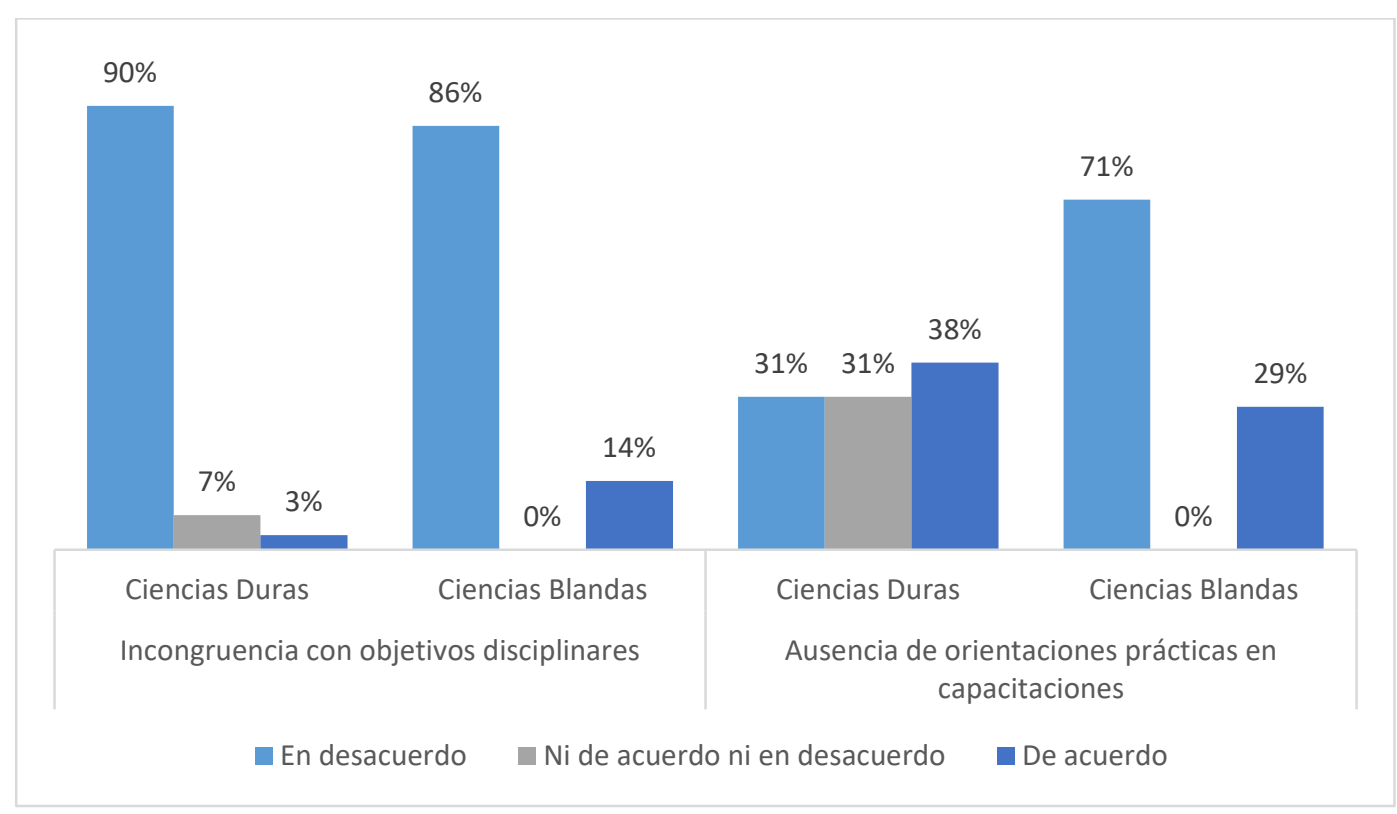

Fuente: Elaboración propia. 
Los resultados también destacan un mayor grado de acuerdo por parte de ambas poblaciones disciplinares con respecto a ciertas implicaciones negativas de tipo institucional (ver Figura 5). Tanto en ciencias duras como en ciencias blandas es mayor la percepción sobre la falta de espacios de colaboración entre docentes.

Figura 5

Percepción de falta de espacios de colaboración e insuficiencia en gestión institucional por disciplinas, Universidad de Sonora-México, 2020

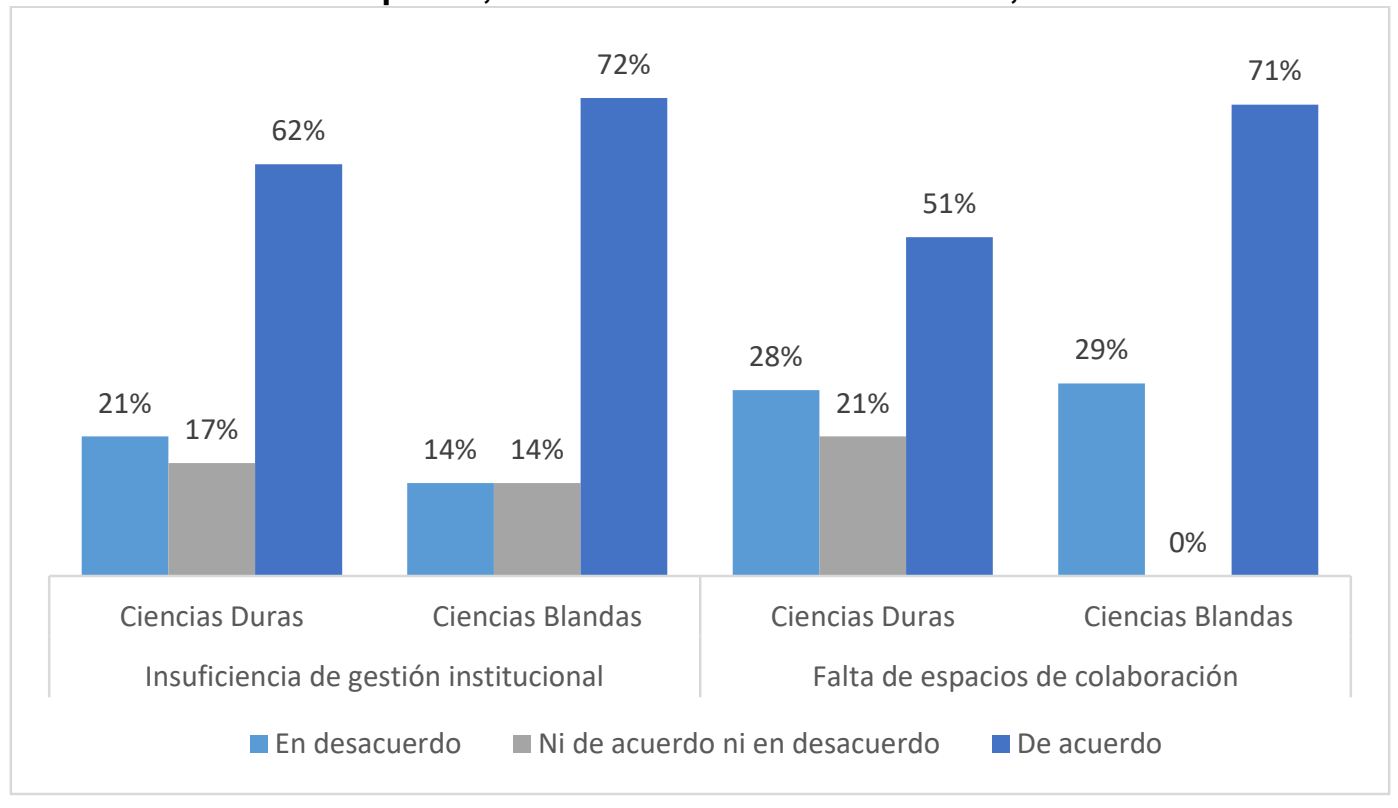

Fuente: Elaboración propia.

De manera sobresaliente, destaca la insuficiencia de la gestión institucional, como la apertura de más asignaturas en inglés en los diferentes departamentos de la universidad, falta de más capacitaciones que les permitan seguir mejorando, por mencionar algunos. El grado de acuerdo fue especialmente mayor en disciplinas de ciencias blandas, en las cuales es menor la cantidad de asignaturas que se imparten en inglés.

Esto se relaciona con lo señalado por Green y Whitsed (2013), quienes exponen a las disciplinas como comunidades con prácticas y necesidades diferentes. Por ende, sus enfoques, requerimientos y necesidades en las tareas de internacionalización del currículo serán diferentes. Así, se alude a la pertinencia de crear espacios interdisciplinarios en donde se compartan diferentes perspectivas y experiencias sobre cómo se afrontan los cambios y retos que implica la impartición de asignaturas con el inglés.

En referencia a sugerencias expresadas por las y los docentes para mejorar esta innovación que propone ofrecer asignaturas en inglés, destaca el mejoramiento de la gestión 
administrativa en los departamentos de la institución (ver Figura 6). También, se advierte la necesidad de un replanteamiento de objetivos de esta implementación, una mejor promoción de las materias con el alumnado, la creación de espacios de colaboración y trabajo, creación de comisiones que den seguimiento a esta iniciativa, además de promover el uso del inglés también de otra forma y en otros espacios.

Figura 6

Porcentaje de las sugerencias del profesorado para mejorar la innovación por tipos de disciplinas, Universidad de Sonora-México, 2020

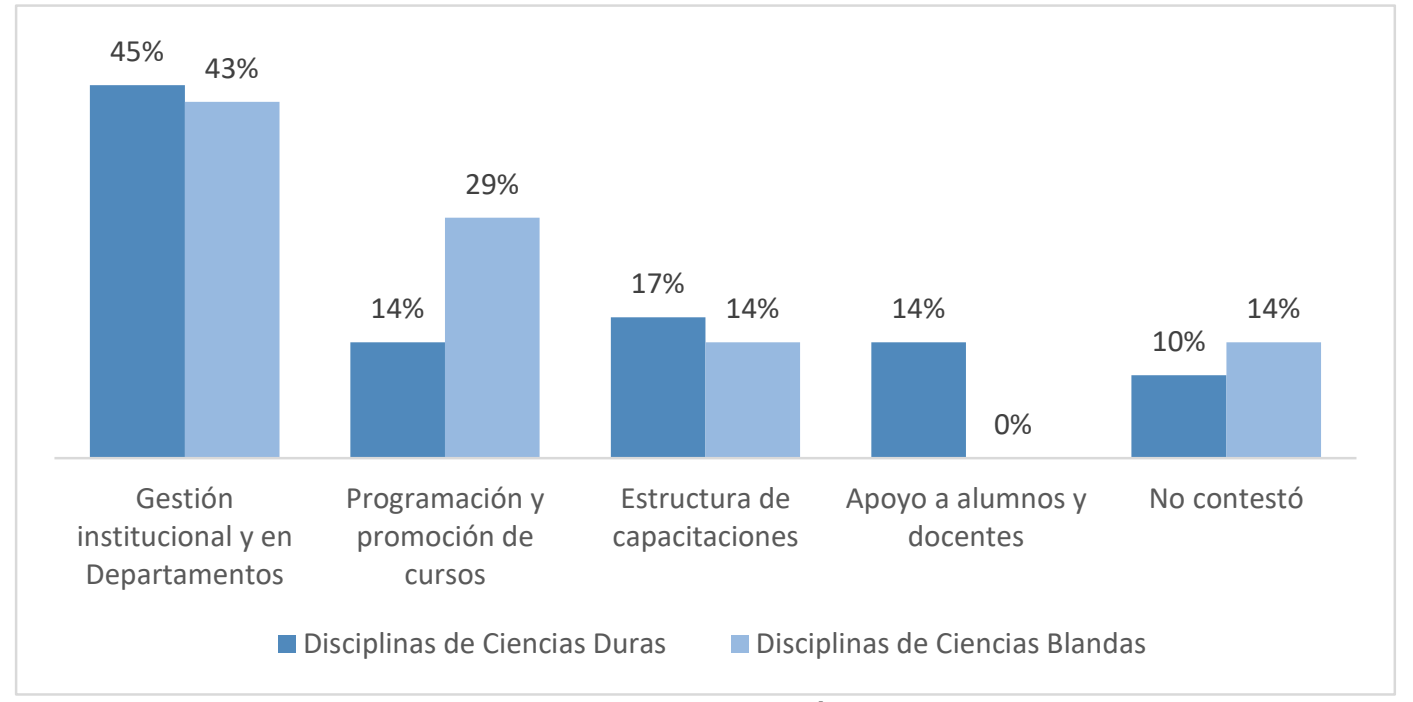

Fuente: Elaboración propia.

En el desglose de estos datos y entre las sugerencias referentes a la gestión institucional, el profesorado destacó como favorable el proceso de capacitación por parte de la institución, pero este esfuerzo se desmerita por los pocos o nulos esfuerzos e interés de diferentes Departamentos por la apertura de grupos en idioma inglés para la inscripción del alumnado. Esto reconoce la necesidad de más apoyo institucional, especialmente en la apertura de espacios para seguir capacitándose, así como para la planeación y mejoramiento de materiales que son útiles en estas asignaturas. Expresan que gran parte del trabajo requerido para estas asignaturas se realiza sin ningún tipo de apoyo ni retroalimentación.

También, resalta la falta de puntos de convergencia entre el personal académico acerca de cuál es el objetivo principal de esta acción innovadora. Por esto, sugieren comprender, en primera instancia, si este tipo de asignaturas atiende las expectativas y posibilidades del alumnado local (del país) o si en realidad es para alumnado del extranjero con la finalidad de 
atracción de más estudiantes de otros países. Esto indica un desconocimiento amplio de lo que se formula para impulsar la internacionalización mediante este tipo de innovaciones.

Con respecto a la programación y promoción de los cursos/asignaturas en inglés, se expresa el obstáculo que representan instancias institucionales encargadas de la internacionalización. Manifiestan que en reiteradas ocasiones solicitan la programación de asignaturas en inglés a las coordinaciones de los programas educativos, sin embargo, perciben un interés nulo de su parte y desconocen las razones de la administración para no ofrecerlas. Además, refieren la importancia de darles mayor difusión entre el alumnado, pero sin olvidar el establecimiento de criterios -como el nivel suficiente del idioma-, al momento de inscribirse a estos cursos.

En lo referente a capacitaciones, se expone la necesidad de continuar con este proceso, además de acceder a un sistema de acompañamiento docente que permita mejorar en el manejo del idioma con fines académicos. También consideran que la estrategia de acreditar docentes a través de un solo curso de larga duración resulta poco efectiva, mientras que la necesidad y posibilidades son diferentes. Esto conlleva a identificar la recomendación de incentivar esta labor, debido al esfuerzo que implica el diseño de una asignatura en inglés, de lo contrario expresan oposición a enseñar en esta modalidad. Para el alumnado, sugieren hacer explícito en su documentación que cursaron una o varias materias en idioma inglés, de forma que al egresar puedan comprobar que poseen la habilidad académica en el idioma.

De esta manera, se reconocen con claridad los retos que la institución enfrenta, además de la necesidad de retomar sus estrategias de tal forma que logren enfocar su atención en las necesidades del personal académico. Esto, a través de una política institucional que, con una mejor estructura, respalde el desarrollo de la docencia con el manejo óptimo del idioma inglés y considere que cada docente inicia una aventura para intentar innovar en su enseñanza.

\section{Conclusiones}

Los resultados encontrados en el contexto particular de la Universidad de SonoraMéxico, evidencian una serie de aspectos que, en su mayoría, restringen el proceso de innovación, referente a la impartición de asignaturas en inglés y, a su vez, la internacionalización. Aunque también se encuentran aspectos alentadores en torno a este proceso.

Por un lado, resalta un interés y disposición por parte del personal académico acreditado para llevar a cabo la innovación con este tipo de enseñanza, a capacitarse para mejorar y 
trabajar con diferentes docentes para la planificación de estas asignaturas. Estos resultados refuerzan y evidencian, en parte, la noción de que este tipo de innovación es compatible con los valores y objetivos de las disciplinas de la universidad y que, debido a esto, las diferentes comunidades docentes comienzan a visualizar los beneficios potenciales de la innovación de enseñanza con el idioma inglés.

Por otro lado, al ser necesario el desarrollo de competencia intercultural para internacionalizar el currículo universitario, la innovación representa un esfuerzo para el profesorado que hace imprescindible la existencia de apoyo institucional en forma de capacitaciones de manera regular, espacios de trabajo colaborativo y retroalimentación para lograr una transformación curricular significativa (Dunne, 2011). En ese sentido, convendría que instituciones que incursionen en este tipo de innovación curricular formen parte del cambio que se busca y requiere, a través de la reflexión y análisis crítico de la práctica que conlleve a generar planes de estudios internacionalizados. Como lo señalan McKinnon et al. (2017), siempre con el respaldo de una implementación de políticas que se ajusten al contexto institucional, con estrategias y acciones que aborden los obstáculos que se deriven. De no ser así, la rentabilidad de la innovación (Rivas, 2000) podría verse afectada. Los resultados evidencian que las y los profesores de ambos tipos de ciencias perciben como compatible el proceso innovador con sus disciplinas y distinguen los potenciales beneficios; no obstante, si los servicios de la universidad fallan en adaptarse a las necesidades individuales (y disciplinares), se configura una situación distante de lograr institucionalizar la innovación y/o el profesorado podría resistirse al cambio al no recibir apoyo.

De modo que, Friesen (2012) plantea que las universidades podrían dejar de centrarse en qué se busca con la internacionalización y enfocarse más en orientar cómo el personal académico pueda dar sentido a este cambio dentro del contexto institucional propio. De otra manera, los alcances de estas propuestas se confinan más en una comprensión programática de la internacionalización. Asimismo, existe la necesidad de introducir pautas más prácticas que orienten la implementación de la internacionalización del currículo (Osakwe, 2017).

Al analizar las sugerencias expuestas por el personal académico, destaca el obstáculo que representa la insuficiente gestión institucional y al interior de las instancias administrativas de los departamentos. Especialmente, por el apoyo limitado e interés inexistente hacia la oferta de asignaturas en inglés, a pesar de existir disposición por parte de docentes. Además de esto, resalta la confusión que existe entre el profesorado que ya fue acreditado sobre los objetivos a alcanzar con este proceso, donde se desconoce si es para mejorar el aprendizaje de 
estudiantes nacionales o para atraer alumnado del extranjero a la institución. Esto coincide con estudios de EMI/CLIL en América Latina, en los cuales también se plantea la necesidad de establecer objetivos más claros y trabajo estratégico en la implementación de dichos enfoques de enseñanza (McDougald, 2015; Corrales et al., 2016). Al implementarse una innovación educativa, es pertinente fomentar una cultura organizacional abierta y de apoyo con metas claras, en la cual se fomente un espíritu colaborativo y una visión compartida (Cai, 2017).

Frente a este panorama analizado, es necesario que los propósitos institucionales sean presentados de manera explícita y clara sobre cómo lograr un plan de estudios internacionalizado y cuáles -y quiénes- son los objetivos por alcanzar, traduciendo su deseo de internacionalización en apoyo práctico para el profesorado (McKinnon et al., 2017). Debido a que, incluso al existir la voluntad para implementar la innovación, Dunne (2011) expone que la disponibilidad de recursos necesarios o el apoyo en forma de guía/retroalimentación para cambiar la pedagogía también representaran un desafío. Esto, se ve plasmado en el caso de la Universidad de Sonora y en otras universidades de México, en concordancia con el estudio de Nuñez Asomoza (2015), el cual también denota áreas de oportunidad en docentes, asuntos administrativos y de gestión institucional referente a programas con EMI/CLIL.

Los resultados de la investigación indican que no se ha manejado un grado de claridad adecuado en el proceso de innovación. Esto, debido a que el profesorado que fue acreditado no distingue sus objetivos, características, ni una estructura institucional bien articulada que los apoye durante la implementación. Esta situación eleva el grado de complejidad de la innovación y hace que sea operativamente difícil para las y los profesores acreditados ofrecer su enseñanza en inglés, al mostrar incertidumbre sobre lo que se busca con este tipo de internacionalización. Además, carecer de instancias dentro de la universidad que apoyen a desarrollar sus capacidades en la práctica.

Al tratarse de cambios en cuestiones pedagógicas y didácticas que evidentemente deben plasmarse en el ejercicio docente, todo tipo de modificaciones resultaran demandantes y en ocasiones abrumadoras. Motivo por el cual la transformación de percepciones hacia la innovación llevará un tiempo más prolongado del planificado (Casanova, 2015). Concretamente, convendría que los programas de capacitación centraran también su atención a los distintos niveles de apropiación que va desarrollando cada docente y que eviten prioritariamente capacitaciones técnicas cortas en tiempo. Las capacitaciones solo aportarán algo cuando se presenten y discutan experiencias significativas, por lo que convendría evitar 
habilitaciones técnicas cortas en tiempo y el dejar al profesorado solo ante las innovaciones (Díaz-Barriga, 2010).

A pesar de los esfuerzos de formación por parte de la institución para la impartición de estas asignaturas, queda desdibujado el establecimiento de objetivos que se buscan para el personal académico desde un abordaje técnico en una capacitación que funcionó como la única herramienta para impulsar la innovación. Si bien, se lograron avances en la implementación de asignaturas en inglés, se comienzan a distinguir inconformidades y pequeñas resistencias a esta innovación que, como lo advierte Rivas (2000), si no son abordadas de manera oportuna podrían generar un rechazo hacia la innovación.

En ese sentido, no menos importante es distinguir la relevancia del seguimiento de toda acción innovadora desde la percepción de las personas involucradas, en especial contemplando aquellas intenciones que buscan promover la internacionalización del currículo mediante la implementación de asignaturas en inglés. Se requiere una reflexión acerca del buen funcionamiento de la enseñanza y el uso de idiomas con fines académicos, siempre acorde a los propósitos y posibilidades institucionales. Como se resalta a partir de los hallazgos, un obstáculo importante para la internacionalización es la estructura de la propia universidad, la cual posiblemente se aleja de generar un entorno que favorezca un cambio significativo y que obstruye la creación de una coordinación centralizada para el seguimiento de la innovación (Childress, 2009). Una limitación importante del estudio es que se trata de una mirada global del fenómeno innovador que tiene lugar en la Universidad de Sonora. Sin embargo, como se distingue en los resultados, la percepción docente varía de forma significativa entre las disciplinas. De modo que, convendría que futuros estudios ahonden en los casos particulares de cada una de las disciplinas con el fin de conocer sus prácticas internacionales, así como sus motivos y necesidades para internacionalizar sus currículos. Esto con el fin de lograr una mejor comprensión de cómo los procesos innovadores que buscan impulsar la internacionalización del currículo en la universidad impactan en las disciplinas y con esto lograr que tengan efecto de manera óptima en los diversos currículos.

Asimismo, convendría enfocar la atención a otros actores que también están involucrados en este proceso de innovación: las instancias administrativas institucionales y el alumnado. Por un lado, la institución y la gestión que lleve a cabo juegan un papel vital como lo expresa el profesorado en el estudio. Desde una mirada política podrían analizarse los costos, beneficios y dificultades que enfrenta la institución al tratar de impulsar la innovación de asignaturas en inglés. Por otro lado, la innovación tendrá efecto en el currículo y en la 
internacionalización de la universidad según el grado en que se fomenta el desarrollo de competencia intercultural en estudiantes. Los resultados evidencian que buena parte de las y los docentes tienen dificultades y dudas sobre cómo trabajar dicha competencia, por lo que sería pertinente indagar en la percepción estudiantil. Esto, con el fin de determinar el impacto que la innovación tiene en su proceso de aprendizaje, la relevancia que le otorgan a la enseñanza con el idioma inglés y las dificultades a las que se enfrentan.

Por último, al tratarse de un primer acercamiento hacia este tipo de internacionalización curricular, el cual se abordó desde la perspectiva de la innovación educativa con el fin de denotar avances y/u obstáculos en el proceso, es pertinente que futuras investigaciones se aborden desde otras miradas teóricas que permitan una concepción más completa del mismo proceso. Aunque los resultados de la investigación fueron de un contexto educativo en particular, se espera que esto propicie el desarrollo de investigaciones en diversos escenarios de educación superior en México y América Latina sobre otras formas innovadoras que buscan promover cambios para desarrollar la internacionalización de la educación superior.

\section{Referencias}

Agnew, Melanie. (2013). Strategic planning: An examination of the role of disciplines in sustaining internationalization of the university. Journal of Studies in International Education, 17(2), 183-202. doi: https://doi.org/10.1177\%2F1028315312464655

Arango, Ana María., y Acuña, Luis Eduardo. (2018). La Internacionalización del currículo y su relación con las condiciones de calidad en los programas académicos de educación superior para la obtención de registro calificado. Revista ObIES, 2, 35-49. Recuperado de https://bit.ly/2HylLWv

Becher, Tony. (1989). Academic tribes and territories: Intellectual enquiry and the cultures of the disciplines. Milton Keynes, United Kingdom: Society for Research into Higher Education and the Open University Press. Recuperado de https://bit.ly/39jzYDg

Bedenlier, Svenja., y Zawacki-Richter, Olaf. (2015). Internationalization of higher education and the impacts on academic faculty members. Research in Comparative \& International Education, 10(2), 185-201. Recuperado de https://bit.ly/35YMQN0

Beelen, Jos. (2011). Internationalisation at home in a global perspective: A critical survey of the $3^{\text {rd }}$ Global Survey Report of IAU. International Journal of Educational Technology in Higher Education, 8(2), 249-264. Recuperado de https://bit.ly/374wwd0

Bell, Maureen. (2004). Internationalising the higher education curriculum: Do academics agree?. En Proceedings of the 27th HERDSA Annual Conference. Congreso llevado a cabo en Miri, Malasia. Recuperado de https://www.herdsa.org.au/publications/conference-proceedings/research-anddevelopment-higher-education-transforming-3 
Bradford, Annette. (2012). Challenges in adopting English-taught degree programs. International Higher Education, (69), 1-6. doi: https://doi.org/10.6017/ihe.2012.69.8646

Cai, Yuzhuo. (2017). From an analytical framework for understanding the innovation process in higher education to an emerging research field of innovations in higher education. Review of Higher Education, 40(4), 585-616. Recuperado de https://bit.ly/3irdJxC

Casanova, María Antonia. (2015). Diseño curricular e innovación educativa. Madrid, España: Editorial La Muralla.

Childress, Lisa. (2009). Internationalization plans for higher education institutions. Journal of Studies in International Education, 13(3), 289-309. doi: https://doi.org/10.1177\%2F1028315308329804

Chostelidou, Dora., y Griva, Eleni. (2014). Measuring the effect of implementing CLIL in higher education: An experimental research project. Procedia-Social and Behavioral Sciences, 116, 2169-2174. doi: https://doi.org/10.1016/j.sbspro.2014.01.538

Clifford, Valerie Anne. (2009). Engaging the disciplines in internationalising the curriculum. International Journal for Academic Development, 14(2), 133-143. Recuperado de https://bit.ly/3li6XKu

Cogo Alessia., y Dewey Martin. (2012). Analysing English as a lingua franca: A corpus-driven investigation. New York, Estados Unidos: Bloomsbury Publishing.

Coleman, James. (2006). English-medium teaching in European higher education. Language teaching, 39(1), 1-14. doi: https://doi.org/10.1017/S026144480600320X

Corrales, Kathleen Anne., Paba Rey, Lourdes., y Santiago Escamilla, Nazira. (2016). Is EMI enough? Perceptions from university professors and students. Latin American Journal of Content \& Language Integrated Learning, 9(2). doi: https://doi.org/10.5294/7094

Coyle, Do. (2010). Content and language integrated learning Motivating learners and teachers. En Do Coyle, Philip Hood y David Marsh, Content and language integrated learning (pp. 1-18). Alemania: Ernst Klett Sprachen. Recuperado de https://scilt.org.uk/Portals/24/Library/slr/issues/13/SLR13 Coyle.pdf

De la Herrán, Agustín y Paredes, Joaquín. (2012). Promover el cambio pedagógico en la universidad. España: Ediciones Pirámide.

De la Torre, Saturnino. (1997). Innovación educativa: El proceso de innovación. España: Dykinson.

De Wit, Hans. (2002). Internationalization of higher education in the united states of America and Europe: A historical, comparative and conceptual analysis. Westport, Estados Unidos: Greenwood Press. 
Delgado-Márquez, Blanca., Hurtado-Torres, Nuria Esther., y Bondar, Yaroslava. (2011). La internacionalización en la enseñanza superior: investigación teórica y empírica sobre su influencia en las clasificaciones de las instituciones universitarias. Universities and Knowledge Society Journal, 8(2), 101-122. Recuperado de https://bit.ly/3629Af7

Díaz-Barriga, Frida. (2010). Los profesores ante las innovaciones curriculares. Revista Iberoamericana de Educación Superior, 1(1), 37-57. Recuperado de https://bit.ly/2KEvPzu

Didou, Sylvie. (2006). Internacionalización de la educación superior y provisión transnacional de servicios educativos en América Latina: del voluntarismo a las elecciones estratégicas. En: Informe sobre la Educación Superior en América Latina y el Caribe 2000-2005 (pp. 22-32). Caracas, Venezuela. Recuperado de https://bit.ly/3lbuxbX

Didou, Sylvie. (2017). La internacionalización de la educación superior en América Latina: transitar de lo exógeno a lo endógeno. Ciudad de México, México: UDUAL. Recuperado de https://bit.ly/39hqVTd

Dunne, Ciarán. (2011). Developing an intercultural curriculum within the context of the internationalisation of higher education: Terminology, typologies and power. Higher Education Research \& Development, 30(5), 609-622. doi: http://dx.doi.org/10.1080/07294360.2011.598451

Escudero, Juan. (2014). Avances y retos en la promoción de la innovación en los centros educativos. Educar, 101-138. Recuperado de https://bit.ly/2UZHNpi

Fàbregues, Sergi, Meneses, Julio, Rodríguez-Gómez, David y Paré, Marie-Helene. (2016). Técnicas de investigación social y educativa. Catalunya, España: Editorial UOC.

Friesen, Rhonda. (2012). Faculty member engagement in Canadian university internationalization: A consideration of understanding, motivations and rationales. Journal of Studies in International Education, 17(3), 209-227. doi: https://doi.org/10.1177\%2F1028315312451132

González, Edgar. (2016). Internacionalización de la educación superior en Sonora, México: un acercamiento inicial. Revista Brasileira de Ensino Superior, 2(1), 41-51. doi: https://doi.org/10.18256/2447-3944/rebes.v2n1p41-51

Green, Wendy y Whitsed, Craig. (2013). Reflections on an alternative approach to continuing professional learning for internationalization of the curriculum across disciplines. Journal of Studies in International Education, 17(2), 148-164. doi: https://doi.org/10.1177\%2F1028315312463825

Jenkins, Jennifer. (2014). The internationalisation of higher education. But what about its lingua franca?. En Waseda Working Papers in ELF (pp. 15-31). Recuperado de https://bit.ly/3IYrrZQ

Khan, Mohammad, Omrane, Amina y Bank, Diana. (2016). The role of internationalization in the higher education industry: an exploratory study. International Journal of Economics \& Strategic Management of Business Process, 7(2), 86-99. Recuperado de http://ipcoco.com/ESMB/ESMB/2.pdf 
Knight, Jane. (1994). Internationalization: Elements and Checkpoints. Canadian Bureau for International Education, $\quad 7 . \quad$ Recuperado de https://eric.ed.gov/?q=Internationalization\%3a+Elements+and+Checkpoints\&id=ED549

Knight, Jane. (1997). Internationalization of Higher Education: A Conceptual Framework. En: J. Knight y $\mathrm{H}$. de Wit (Eds), Internationalization of Higher Education in the Asia Pacific Countries (pp. 5-19). Amsterdam: European Association of International Education (EAIE).

Knight, Jane. (2008). Higher Education in Turmoil. The Changing World of Internationalization. Rotterdam, Países Bajos: Sense Publishers.

Knight, Jane., y De Wit, Hans. (1995). Strategies for internationalisation of higher education: Historical and conceptual perspectives. En Strategies for internationalisation of higher education: A comparative study of Australia, Canada, Europe and the United States of America. Recuperado de https://bit.ly/3fuxd2Q

Leask, Betty. (2001). Bridging the gap: Internationalizing university curricula. Journal of Studies in International Education, 5(2), 100-115. doi: https://doi.org/10.1177\%2F102831530152002

Marsh, David., Pavón-Vázquez, Víctor., y Frigols-Martín, María Jesús. (2013). The higher education languages landscape: Ensuring quality in English language degree programmes. Valencia, Spain: Valencian International University. Recuperado de https://bit.ly/3o00825

McDougald, Jermaine. (2015). Teachers' attitudes, perceptions and experiences in CLIL: A look at content and language. Colombian Applied Linguistics Journal, 17(1), 25-41. doi: http://dx.doi.org/10.14483/udistrital.jour.calj.2015.1.a02

McKinnon, Sabine., Hammond, Angela., y Foster, Monika. (2017). Reflecting on the value of resources for internationalizing the curriculum: exploring academic perspectives. Journal of Further and Higher Education, 43(1). doi: https://doi.org/10.1080/0309877X.2017.1359506

McMillan, James., y Schumacher, Sally. (2005). Investigación educativa (5 $5^{\mathrm{a}}$ ed.). Madrid: Pearson Addison Wesly.

Nikula, Tarja. (2016). CLIL: A European approach to bilingual education. En N.V. DeusenScholl y S. May (Eds.), Encyclopedia of Language and Education (pp. 1-13), Springer. Recuperado de https://bit.ly/2UZKbwg

Nilsson, Bengt. (2003). Internationalisation at home from a Swedish perspective: The case of Malmö. Journal of Studies in International Education, 7(1), 27-40. doi: https://doi.org/10.1177\%2F1028315302250178

Nuñez Asomoza, Alejandra. (2015). Students' perceptions of the impact of CLIL in a Mexican BA program. PROFILE Issues in Teachers' Professional Development, 17(2), 111-124. doi: http://dx.doi.org/10.15446/profile.v17n2.47065 
Osakwe Nneka, Nora. (2017). Internationalizing Courses: A Faculty Development Process. International Research and Review, 6(2), 1-31. Recuperado de https://bit.ly/39sbQOK

Paricio, María Silvina. (2014). Competencia intercultural en la enseñanza de lenguas extranjeras. Porta Linguarum (21), 215-226. Recuperado de https://bit.ly/3fx90ch

Rivas, Manuel. (2000). Innovación educativa: teoría, procesos y estrategias. Madrid, España: Síntesis.

Rodríguez, David., y Valldeoriola, Jordi. (2009). Metodología de la investigación. España: Universitat Oberta de Catalunya.

Smit, Ute., y Dafouz Emma. (2012). Integrating content and language in higher education. An introduction to, English-medium policies, conceptual issues, and research practices across Europe. AILA Review 25(1), 1-12. Recuperado de https://www.researchgate.net/publication/263341106 Integrating content and languag $\mathrm{e}$ in higher education An introduction to Englishmedium policies conceptual issues and research practices across Europe

Tejada, José. (1998). Los agentes de la innovación en los centros educativos. Profesores, directivos y asesores. Málaga, España: Aljibe.

Turner, Yvonne., y Robson, Sue. (2007). Competitive and cooperative impulses to internationalization: Reflecting on the interplay between management intentions and the experience of academics in a British university. Education, Knowledge \& Economy, 1(1), 65-82. Recuperado de: https://bit.ly/3uhHKp2

Van der Wende, Marijk. (1996). Internationalizing the curriculum in higher education: Report on a OECD/CERI study. Tertiary Education and Management, 2(2), 186-195. doi: https://doi.org/10.1080/13583883.1996.9966900

Van der Wende, Marijk. (1999). An innovation perspective on internationalation of higher education institutionalisation: The critical phase. Journal of Studies in International Education, 3(1), 3-14. doi: https://doi.org/10.1177\%2F102831539900300102

Wächter, Bernd y Maiworm, Friedhelm (Eds.). (2014). English-taught programmes in European higher education: The State of Play in 2014. Bonn, Alemania: Lemmens. Recuperado de https://bit.ly/377Xa4y

Wihlborg, Monne. (2009). The pedagogical dimension of internationalisation? A challenging quality issue in higher education for the twenty-first century. European Educational Research Journal, 8(1), 117-132. doi: http://dx.doi.org/10.2304/eeri.2009.8.1.117 


\section{Anexos}

Anexo 1: Cuestionario Iniciativa innovadora de asignaturas impartidas en idioma inglés para la internacionalización del currículo

\begin{tabular}{|c|c|c|c|c|c|}
\hline \multirow{2}{*}{\multicolumn{6}{|c|}{$\begin{array}{l}\text { Preguntas a docentes acreditados para asignaturas en inglés de la Universidad de } \\
\text { Sonora }\end{array}$}} \\
\hline & & & & & \\
\hline \multicolumn{6}{|c|}{ El uso del idioma inglés en clase tiene las implicaciones negativas siguientes: } \\
\hline & 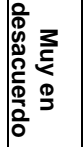 & & & & 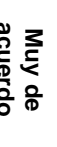 \\
\hline & 1 & 2 & 3 & 4 & 5 \\
\hline \multicolumn{6}{|l|}{ 1. Acceso insuficiente a recursos y materiales en este idioma } \\
\hline \multicolumn{6}{|l|}{ 2. Incongruencia entre los objetivos disciplinares y el uso de inglés en clase } \\
\hline \multicolumn{6}{|l|}{ 3. Dominio insuficiente del idioma por el estudiante } \\
\hline \multicolumn{6}{|l|}{ 4. Incrementa el tiempo de dedicación a mis actividades académicas } \\
\hline \multicolumn{6}{|l|}{$\begin{array}{l}\text { 5. Requiero apoyo y retroalimentación para llevar a cabo mi práctica de } \\
\text { enseñanza con el idioma }\end{array}$} \\
\hline \multicolumn{6}{|l|}{$\begin{array}{l}\text { 6. Requiero mejorar mis habilidades para adaptar los contenidos y } \\
\text { materiales en inglés. }\end{array}$} \\
\hline \multicolumn{6}{|l|}{$\begin{array}{l}\text { 7. Requiero aprender cómo incluir, trabajar y evaluar la competencia } \\
\text { intercultural en clase }\end{array}$} \\
\hline \multicolumn{6}{|l|}{$\begin{array}{l}\text { 8. Faltan espacios de discusión y colaboración para planear estas } \\
\text { asignaturas. }\end{array}$} \\
\hline \multicolumn{6}{|l|}{$\begin{array}{l}\text { 9. Ausencia de orientaciones prácticas desde las capacitaciones para la } \\
\text { enseñanza en inglés. }\end{array}$} \\
\hline $\begin{array}{l}\text { 10. Insuficiencia de la gestión institucional para lo que requieren estas } \\
\text { asignaturas }\end{array}$ & & & & & \\
\hline
\end{tabular}


Revista indizada en

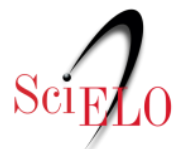

Distribuida en las bases de datos:

- Dialnet

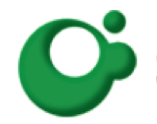
SHERPA/RoMEO

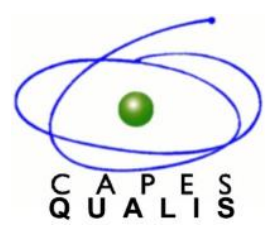

MIAR 